\title{
CAN REFLECTION FROM GRAINS DIAGNOSE THE ALBEDO?
}

\author{
John S. Mathis, ${ }^{1}$ Barbara A. Whitney, ${ }^{2}$ And Kenneth Wood ${ }^{3}$ \\ Received 2002 February 27; accepted 2002 April 3
}

\begin{abstract}
By radiation transfer models we show that the optical properties of grains are poorly constrained by observations of reflection nebulae. The interstellar medium is known to be hierarchically clumped, from a variety of observations (molecules, H I, far-infrared). We have performed radiative transfer through four-tiered, hierarchically clumped dust in a sphere surrounding a central star. Our models have realistic power spectra of the projected density distributions (index $\sim-3$ ). The input parameters are the albedo $(a)$ and phase parameter $(g)$ of the dust, the radial optical depth of the sphere averaged over all directions $\left(\tau_{0}\right)$, and the detailed random distribution of the dust clumps within the sphere. The outputs are the stellar extinction, optical depth, and flux of scattered light as seen from various viewing angles. Observations of a reflection nebula provide the extinction and scattered flux as viewed from one particular direction. Hierarchical geometry has a large effect on the flux of scattered light emerging from a nebula for a particular extinction of the exciting star. There is a very large spread in both scattered fluxes and stellar extinctions for any distribution of dust. Consequently, an observed $\left(\tau_{\text {ext }}, \tau_{\text {sca }}\right)$ can be fitted by a wide range of albedos. There are lower limits on $a$ set by the scattered flux. As an example, in the best-observed reflection nebula, NGC 7023, $a(1300 \AA)$ must be higher than $\sim 0.5$ if the scattered flux from Witt et al. and a reasonable value for the optical depth within the nebula are adopted. However, the same observations can be fitted with $a=0.8$ and $0.6 \leq g \leq 0.85$, the entire range we considered. With hierarchical geometry it is not completely safe to determine even relative optical constants from multiwavelength observations of the same reflection nebula. The problem is that the geometry effectively changes with wavelength as the opacity of the clumps varies. Limits on the implications of observing the same object in various wavelengths are discussed briefly. Henry uses a recipe to determine the scattered flux from a star with a given extinction. It is claimed to be independent of the geometry. It provides considerably more scattering for given dust optical properties than our models, probably leading to an underestimate of the grain albedos from the UV diffuse Galactic light.
\end{abstract}

Subject headings: dust, extinction — reflection nebulae — scattering

\section{INTRODUCTION}

Interstellar dust is poorly understood, in spite of its importance within galaxies. The mean extinction law (i.e., scattering plus absorption) for the diffuse Galactic interstellar medium (ISM) is reasonably well known, but the individual absorption and scattering properties are still controversial. Many other basic properties (chemical composition, particle size distribution, particle shape distribution, alignment of the grains in the magnetic field, and others) are also poorly known.

The properties of dust for each scattering event are characterized by two parameters: $a(\lambda)$, the albedo or fraction of the extinction that is scattering, and $g(\lambda)$, the mean cosine of the scattering angle, averaged over the phase function for single scattering. Geometrical uncertainties prevent the determination of higher moments of the scattering pattern beyond these two. The $a$ and $g$ are estimated from either reflection nebulae excited by a central star, radiation from a dusty globule reflecting Galactic light, or the "diffuse Galactic light" (DGL), the general stellar radiation reflected by the dust distributed throughout the ISM.

\footnotetext{
${ }^{1}$ University of Wisconsin-Madison, 475 North Charter Street, Madison, WI 53706; mathis@astro.wisc.edu.

${ }^{2}$ Space Science Institute, 3100 Marine Street, Suite A353, Boulder, CO 80303; bwhitney@colorado.edu.

${ }^{3}$ School of Physics and Astronomy, University of St. Andrews, North Haugh, St. Andrews, Fife KY169AD, Scotland, UK; kw25@, st-andrews.ac.uk.
}

Almost always, nebulae have been interpreted with smooth (nonclumpy) models, sometimes with a density gradient. Radiation transfer differs in smooth and clumpy media because photons can escape between the clumps. Uniformity was a reasonable assumption, for three reasons. (1) It is the simplest, and Occam's Razor has great appeal. (2) Hierarchical clumping had not been convincingly demonstrated by several means. (3) The computational resources required for radiative transport through hierarchical clumps have only recently become widely available.

Any estimate of the albedo is affected by the hierarchical clumping of the ISM. These clumps occur on scales from 1 $\mathrm{AU}$ to tens or hundreds of parsecs, as observed in various molecules $(\mathrm{CO}$, formaldehyde, and $\mathrm{OH})$, ionic species, $\mathrm{H} \mathrm{I}$, and $100 \mu \mathrm{m}$ (Crovisier \& Dickey 1983; Green 1993; Moore \& Marscher 1995; Vogelaar \& Wakker 1994; Elmegreen \& Falgarone 1996; Abergel et al. 1996; Elmegreen 1997; Heiles 1997; Heithausen et al. 1998; Falgarone et al. 1998; Chappell \& Scalo 2001; Welty \& Fitzpatrick 2001; Faison \& Goss 2001; Andrews, Meyer, \& Lauroesch 2001). Other galaxies (Stanimirović et al. 1999 for the SMC; Westpfahl et al. 1999 for the M81 group) show the same phenomena in $\mathrm{H}$. The best-observed reflection nebulae are clumpy (see, e.g., Sellgren, Werner, \& Dinerstein 1992; Martini, Sellgren, \& DePoy 1999; Knauth et al. 2001). We have examined the images in the SIMBAD database of the reflection nebulae with which we are acquainted (about a dozen); all appear to be clumpy. The observed thermal pressure in the ISM, as judged from C I (Jenkins \& Tripp 2001), varies by over an 
order of magnitude within a sample of stars against which the column densities of $\mathrm{C}$ I lines can be determined. The hierarchical structure of the ISM is a natural consequence of turbulence, which is scale-free. Turbulent models of the ISM generate hierarchical density structure (see, e.g., Norman \& Ferrara 1996).

Boissé (1990) determined the transport of radiation through a two-phase, clumpy medium with isotropic scattering. Witt \& Gordon (1996) extended the calculations to anisotropic scattering in a centrally illuminated sphere. They investigated the effects of changing various parameters, such as the density contrast between the phases or the sizes of the clumps relative to the radius of the sphere. Many of the qualitative effects we find from varying parameters for hierarchical models are discussed by them as well. As the volume fraction of the dense phase was increased, their models varied from widely separated dense clumps up to continuous structures with small holes. Since all cells have either of two densities, we refer to this type of clumping as "two-phase models." By contrast, our hierarchical models have an almost continuous distribution of densities.

Witt \& Gordon (2000) have investigated radiative transfer in galaxies by means of a specific two-phase ISM, one phase being 100 times as dense as the other and occupying $15 \%$ of the volume. In order to compare two-phase with hierarchical models, we adopt this recipe, with 16 cells along the radius of the sphere (Witt \& Gordon used 15). All of our two-phase models assumed $(a, g)=(0.6,0.6)$, which are values typical for optical wavelengths suggested by observations (see figures in Witt \& Gordon 2000).

The fact that the ISM is hierarchically clumped is important for the propagation of scattered light, since such a structure has relatively open spaces through which the radiation can move rather freely. The main thrust of this paper is not to try to put limits on the grain properties of real reflection nebulae, but to show the variation among hierarchical models as they are viewed from various angles. We are not very concerned with the averaged properties of the models, since each real object is viewed from only one direction.

\section{HIERARCHICALLY CLUMPED MODELS}

The density structure of the ISM is often described as "fractal," meaning self-similar on all size scales. We consider models that are hierarchically clumped instead, meaning that they are self-similar over a limited range (about a factor of 10) in sizes. We use a procedure similar to that in Elmegreen (1997). (1) Consider a "supercube," a portion of which will represent a spherical reflection nebula. The supercube, of size $L$ on a side, consists of 64 cubical cells stacked along each dimension. For each cell we determine the local density of dust, as explained below. (2) Place $N$ points randomly within the supercube. We used $N=32$. (3) Randomly cast another $N$ points, all within a distance $L /(2 \Delta)$ in each Cartesian axis from each of the points cast in the preceding round. Here the distance $\Delta$ is related to the "fractal dimension" $D$ by $D \equiv \log (N) / \log (\Delta)$. Allow any of the points that fall outside of the supercube to remain there. (4) Repeat procedure 3 twice more, so that the total number of points cast is $N^{4}$. (5) Shift the points outside the supercube to within it by translating each Cartesian coordinate outside the supercube by $L$ until it lies within. This procedure reflects the points, so that if they originally fall off the left side of the supercube they reappear the same distance from the right within the supercube, and correspondingly for up/down and front/back. The density within the supercube is then proportional to the number of points within each cell. (6) Inscribe a sphere within the supercube and place a point source of radiation at its center. The constant of proportionality between the number of points in each cell and the optical depth within the cell is chosen to make the radial optical depth, averaged over all directions, be $\tau_{0}$.

The main parameter of this procedure is $D$, which describes the degree of clumping. We considered $D=2.3$ and 2.6, in the range observed in actual clouds. Another observable parameter is $\beta$, the exponent of the power of the projection of the density within the supercube onto the plane of the sky, ${ }^{4}$ so that $P(k) \propto k^{-\beta}$, where $k$ is the wavenumber. Observations (see, e.g., Stanimirović et al. 1999) show $\beta \sim 3$. With our recipe we find $\beta=2.8$ with $D=2.6$ and $\beta \sim 2.3$ with $D=2.3$. However, none of the conclusions in this paper depend upon which set of hierarchical models we consider. Many different models can have the same $\beta$. No one parameter, either $D$ or $\beta$, can completely describe even the projection of the density distribution of the ISM onto the sky.

The above procedure, with the parameters we adopted, results in clouds that are quite full of holes, with $\sim 15 \%$ of the projected density distribution being almost blank. The large-scale ISM, as shown by $\mathrm{H}$ I, is similar if a low column density of $\mathrm{H} \mathrm{I}$ (less than $5 \times 10^{19} \mathrm{H}$ atoms $\mathrm{cm}^{-2}$ ) is taken to be "blank" (i.e., a column density much smaller than the average). Elmegreen (1997) has discussed the emptiness of the ISM, with the large-scale structure in mind. Images of actual reflection nebulae (see Sellgren et al. 1992 for NGC 2023 and NGC 7023) show strong filaments and structure, but there is material at all points within the nebula. For this reason, we sometimes add a uniform density to all cells in the supercube. This constant density does not change the value of $\beta$, since the Fourier transform of a constant is a Dirac $\delta$-function at the origin, $k=0$. Choosing a constant density provides a minimum projected density.

The detailed placement of the clumps of dust also influences the results of our radiative transfer calculations. The clumping is mainly formed by the first round of random casting of 32 points, which are subsequently spread by three more rounds of casting points in their vicinities. The locations of the first round of points is uniquely determined by the integer seed of the random number generator, ${ }^{5}$ and the locations of all subsequent points follow from the initial value of this integer in a complicated but unique way. The response of the model nebula to the central star is strongly affected by the precise placement of the dust relative to the star and, thereby, to the value of the initial seed. We considered suites of models that differed only in their initial seeds. Our procedure amounts to assuming that the star does not affect the density distribution of the dust in its immediate vicinity, so the initial seed is allowed to control the placement of the dust both near and far from the star. To test the

\footnotetext{
${ }^{4}$ The structure function $F(\delta \boldsymbol{r})$ is defined by $F(\delta \boldsymbol{r}) \equiv$ $(\text { area })^{-1} \iint I(\boldsymbol{r}) I(\boldsymbol{r}+\delta \boldsymbol{r}) d x d y$, and $P(k)$ is its Fourier transform, with $k=|\delta \boldsymbol{r}|^{-1}$. Here $\boldsymbol{r}$ and $\delta \boldsymbol{r}$ are vectors, and $(x, y)$ Cartesian coordinates, in the plane of the sky.

${ }^{5}$ We used ran2(iseed), as described in Press et al. (1992).
} 
sensitivity of results to this assumption, we also considered models with a cavity in the dust distribution within the inner $10 \%$ of the radius of the sphere.

The radiative transfer was performed by the Monte Carlo code described by Wood \& Reynolds (1999). It involves considering photon packages propagated in a random direction from the central star. We assumed $a$ and $g$, along with the scattering phase function of Henyey \& Greenstein (1941). In most cases we used $5 \times 10^{6}$ photons for each model, after checking for a few cases that the results were the same as from a model using $2 \times 10^{7}$ photons. Unlike the situation for placing the dust, the initial seed for the radiative transfer makes no difference, as expected from such a large number of stellar photons propagated in random directions.

We calculated the fluxes of scattered radiation and also of starlight, both of which depend on the angle from which the sphere is viewed because of the strong randomly placed clumping. Both fluxes are expressed relative to the stellar flux that would be observed from the unobscured star. We express the fluxes as extinction and scattering optical depths, $\quad \tau_{\text {ext }} \equiv-\ln \left(F_{*} / F_{* 0}\right) \quad$ and $\tau_{\text {sca }} \equiv-\ln \left(F_{\text {sca }} / F_{* 0}\right)$, where $F_{* 0}$ is the flux the star would have if there were no reflection nebula. Each flux, or optical depth, depends on the direction from which the sphere is viewed. We considered 18 evenly spaced values of $\cos (\theta)$ and 36 values of $\phi$, where $\theta$ is the polar angle of the sphere and $\phi$ the azimuthal. $F_{\text {sca }}$ and $F_{*}$ can be determined directly (with difficulty!) in real nebulae. $F_{* 0}$ can be determined if there is enough wavelength coverage to determine the extinction from extrapolation to large wavelengths, from colors if the reddening law is assumed, or if the total far-infrared (FIR) flux from the nebula is measured. Otherwise, the ratio of the stellar to the nebular flux gives the difference of $\tau_{\text {ext }}$ and $\tau_{\text {sca }}$. We now ask, how well can we determine $(a, g)$ from an observation of $\left(\tau_{\text {ext }}, \tau_{\text {sca }}\right)$ ?

\section{RESULTS}

As regards radiative transfer, hierarchical clumping introduces major differences from a uniform distribution or, to a lesser extent, from two-phase models. The main properties of hierarchical models are as follows.

1. When viewed from various directions, hierarchically clumped nebulae exhibit $\tau_{\text {ext }}$ and $\tau_{\text {sca }}$ spanning a wide range. A real reflection nebula represents viewing a collection of dust from one particular direction. We can calculate mean properties of models as averages over all viewing directions, but mean properties are relevant only if a large collection of nebulae (such as in another galaxy) are observed. Angleaveraged properties of any model have very limited applicability for interpreting a given reflection nebula. The twophase models of Witt \& Gordon (1996) also show a wide range of $\tau_{\text {ext }}$ at a given $\tau_{0}$.

2. As expected, the global parameters of the models, such as $\tau_{0}$ and $D$, have a significant influence on the radiation transfer. We considered both purely hierarchical models and some with $33 \%$ of the mass in a constant-density sphere upon which the clumps are superimposed. This uniform background density can have an important effect on the radiation transfer in optically thick nebulae, where the diffusion depends more upon the transparent regions than on the opaque. This rule is familiar in connection with the use of the Rosseland mean opacity in the optically thick limit of radiation diffusion in stars. Whether such a uniform layer exists in reflection nebulae is not clear. The regions between clumps on large (molecular cloud) scales have a vastly lower density than the mean (see, e.g., Elmegreen 1997).

3 . Models with the same global parameters $\left(\tau_{0}, D, a\right.$, and $g$ ), but differing in the placement of the dense clumps relative to the central star, show strong variations in even the averaged fluxes. There are significant differences between hierarchical models in which the star happens to lie within a dense clump of dust, and those in which it falls within a void (or for which there is a central hole). If the star happens to lie within a void, there are lightly reddened paths that reach from the star to the edge of the sphere. If the star is almost unattenuated as seen from a particular direction, the nebula would be interpreted as having the star in front of the dust, except that there can be a significant amount of scattered light that would not be there if the star were truly foreground. In these cases, the albedo derived from uniform models would be greater than 1 .

The power-law index, $\beta$, of the projected density distribution of our two-phase models is $\sim 0$, reflecting the fact that their projected density is nonhierarchical. The two-phase models always provide a smaller range in $\tau_{\text {sca }}(\theta, \phi)$ (about \pm 0.12 ) than the hierarchical, but their spread in $\tau_{\text {ext }}$ is comparable to hierarchical models at low $\tau_{0}(\lesssim 2)$. The spread at high $\tau_{0}$ decreases because the interclump medium becomes opaque, so that in the ultraviolet (UV), two-phase models make a prediction of $a$ that is rather independent of viewing angle.

Figure 1 shows $\tau_{\text {sca }}$ plotted against $\tau_{\text {ext }}$ for the case $\left(a, g, D, \tau_{0}\right)=(0.6,0.6,2.6,2)$, for a hierarchical distribution of clumps with an initial seed for the density distribution that provides a typical (defined below) placement with respect to the star. Each point represents a particular viewing direction. One sees a large spread in the values of $\tau_{\text {ext }}$. The values for $\tau_{\text {ext }}$ for this model range up to 6.8 and those for $\tau_{\text {sca }}$ to 2.4. Thus, this one model of clumpy dust can produce a wide range of stellar fluxes and scattered light.

We represent the direction-averaged escaping flux (not mean optical depth) by $\left\langle\tau_{\text {ext }}\right\rangle$, defined by

$$
\exp \left(-\left\langle\tau_{\text {ext }}\right\rangle\right)=\sum_{\theta, \phi} \exp \left[-\tau_{\operatorname{ext}}(\theta, \phi)\right] / N_{\theta, \phi},
$$

where $N_{\theta, \phi}$ is the number of bins we are using $(18 \times 36)$, all of equal solid angle. If the star is embedded within a clump, the uniform dust component is of reduced importance because scattering occurs within the clump. Similarly, $\left\langle\tau_{\text {sca }}\right\rangle$ represents the angle-averaged scattered radiation, with $\tau_{\text {sca }}$ replacing $\tau_{\text {ext }}$ in the above relation. The square in Figure 1 shows $\left\langle\tau_{\text {ext }}\right\rangle$ and $\left\langle\tau_{\text {sca }}\right\rangle$, which are useful for contrasting the results of models with the same value of $\tau_{0}$ but different initial seeds. As the figure shows, the $\left(\left\langle\tau_{\text {ext }}\right\rangle,\left\langle\tau_{\text {sca }}\right\rangle\right)$ point is of very limited value in guessing what actual optical depths a given nebula might show if it were viewed from various angles. In this model $\left\langle\tau_{\text {ext }}\right\rangle$, which describes the average optical depth of the stellar flux that escapes from the nebula, is only $0.5 \tau_{0}$, the averaged optical depth through both the clumps and interclump material. It is sobering to recall that only a handful of reflection nebulae have been well observed, while each point in the figure represents a view of a single model with a particular clumping, $a, g$, and $\tau_{0}$. 


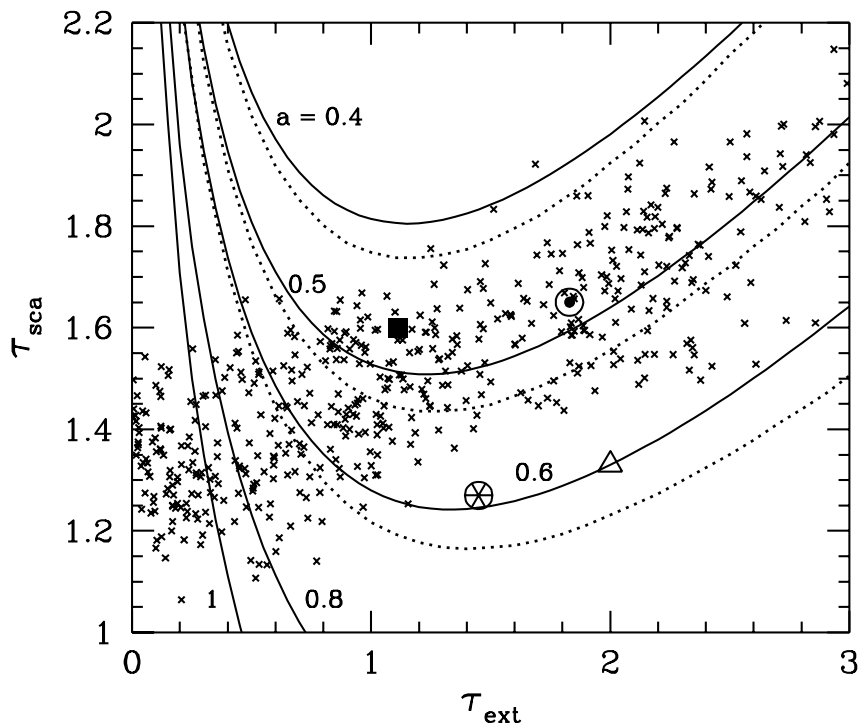

FIG. 1.-Optical depth of scattering in a spherical cloud illuminated by a central star vs. effective optical depth for the stellar radiation. Here $\tau_{\text {sca }} \equiv-\ln \left(F_{\text {sca }} / F_{* 0}\right), F_{\text {sca }}$ is the scattered flux from the nebula, and $F_{* 0}$ is the stellar flux if there were no reflection nebula. Similarly, $\tau_{\mathrm{ext}} \equiv-\ln \left(F_{*} / F_{* 0}\right)$. The crosses represent the fluxes from a hierarchical model with albedo $a=0.6$, phase parameter $g=0.6$, average radial optical depth for the sphere $\tau_{0}=2$, and clumping with a "fractal dimension" $D=2.6$. Each cross represents a direction of viewing the sphere. The square represents $\left(\left\langle\tau_{\text {ext }}\right\rangle,\left\langle\tau_{\text {sca }}\right\rangle\right)$, the fluxes (not optical depths) of the model, averaged over viewing angles (see eq. [1]). We see a very wide range of points for the hierarchical model, as viewed for various directions. The triangle represents the point for a uniform model. The circled asterisk shows the observed point for NGC 7023, a well-observed reflection nebula, at $1300 \AA$ (Witt et al. 1993) if $40 \%$ of the observed extinction toward the central star is in NGC 7023 , and the circled dot the same if $50 \%$ of the extinction is in NGC 7023. Solid lines show the relation for a uniform distribution, with various dust albedos marked. Dotted lines show the relationship assumed by Henry (2002) in analyzing the DGL. Albedos are those indicated at the nearby solid lines.

The solid lines in Figure 1 are the tracks of a spatially uniform dust distribution for the same $g(0.6)$ and various values of $a$, as labeled. These lines were computed with the surprisingly accurate analytical approximation (the scattered flux is underestimated by $\leq 3 \%$ for $\tau_{\text {ext }}<4$ ) given by Code (1973). Most reflection nebulae have been interpreted using these uniform models, in which case $\tau_{\text {ext }}$ is the true optical depth of the nebula. For our hierarchical model, in about $15 \%$ of viewing directions we would conclude that the star is in front of most of the dust because the uniform models would give $a>1$. In $40 \%$ of the directions we would conclude that $a>0.6$, the value assumed in the hierarchical model.

Two UV observations of NGC 7023 are shown in the figure. The central star, HD 200775, has foreground extinction from ordinary ISM in addition to the extinction provided by the nebula (see Witt et al. 1993 for discussion and other references to observations of NGC 7023). The circled asterisk marks the nebular parameters derived from Voyager 2 and Hopkins Ultraviolet Telescope (HUT) data if $40 \%$ of the total extinction arises within the nebula, and the circled dot those if the nebula provides $50 \%$. The dashed lines are discussed in $\S 4.2$, in connection with the UV DGL.

Figure 2 shows the same model as Figure 1, except $33 \%$ of the dust is in uniform distribution, upon which the hierarchical clumping is superimposed. The axes of the figures are

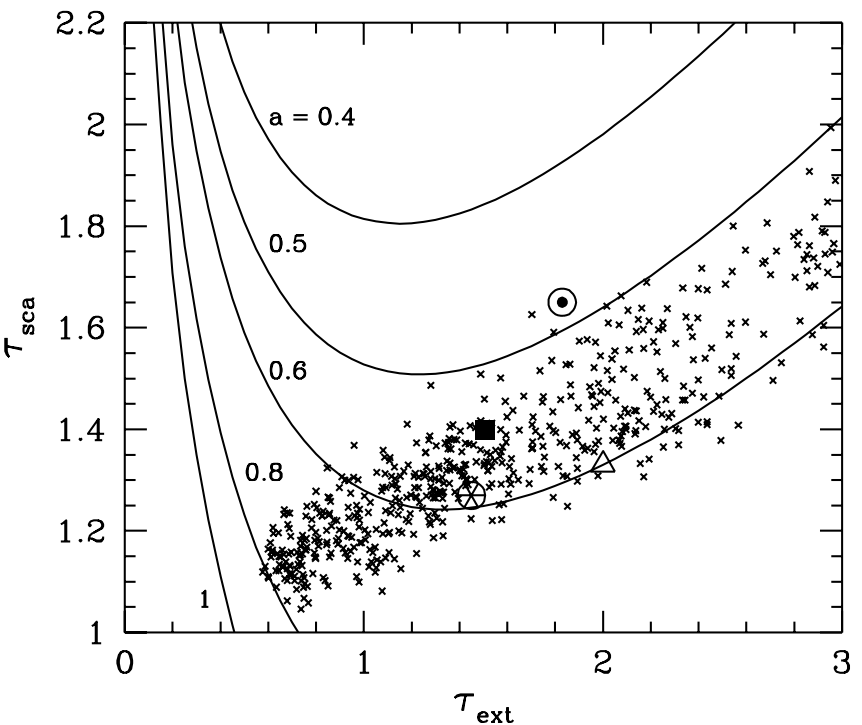

FIG. 2.- Same as Fig. 1, except that $33 \%$ of the dust density is assumed to be in a uniform density underlying the hierarchical clumps. The $\tau_{\text {sca }}$ points are lower than in Fig. 1, showing more scattered light.

the same as in Figure 1, to emphasize that a uniform density increases the scattered light, so long as its optical depth along a radius is not too large $(\lesssim 1)$, and imposes a minimum value of $\tau_{\text {ext }}$. The range of $\tau_{\text {sca }}$ for a given $\tau_{\text {ext }}$ is smaller than in Figure 1, but this is an artifact of the uniformity of the extra component that is the only difference between the two figures.

Wide areas of the $\left(\tau_{\text {ext }}, \tau_{\text {sca }}\right)$ plane are covered by each hierarchical model, so a given $\left(\tau_{\text {ext }}, \tau_{\text {sca }}\right)$ observation can be covered by a wide variety of models. By contrast, uniform models have very limited dependence on $g$ and so contain only one effective parameter, $a$. The ambiguity of hierarchical models is suggested by Figure 3. It has the same axes as Figures 1 and 2, but only outlines around the areas covered by the points of five hierarchical models. The solid line represents a high $a(0.8)$, a moderate $g(0.6)$, and a low $\tau_{0}(0.5)$. The maximum values of $\tau_{\mathrm{ext}}$ are comparatively small because of the low $\tau_{0}$. The dashed line encloses the points for the same model with $33 \%$ of the dust mass in a constant density; the scattered points are lower (there is more scattering) because there are no almost empty paths through the nebula. The dot-dashed and dotted lines are the boundaries of the points in Figures 1 and 2, which are for $\left(a, g, D, \tau_{0}\right)=(0.6,0.6,2.6,2)$. The longdashed lines enclose the very wide boundaries for $\left(a, g, D, \tau_{0}\right)=(0.8,0.85,2.3,4)$. We can have high or moderate $g$, high or moderate $a$, and high or low $\tau_{0}$ and still fit the observations. However, we see in $\S 4.1$ that a minimum $a$ of $\sim 0.5$ is required to produce enough scattered light.

Figure 4 shows the averaged $\left(\left\langle\tau_{\text {ext }}\right\rangle,\left\langle\tau_{\text {sca }}\right\rangle\right)$ values for all of the 21 different initial seeds that we tried. Figure $4 a$ shows purely hierarchical models, Figure $4 b$ models with $33 \%$ of the dust in a constant distribution. The parameters are the same as in Figures 1 and 2: $\left(a, g, \tau_{0}\right)=(0.6,0.6,2)$. The NGC 7023 points are also the same. The open squares are models with $D=2.3$, instead of 2.6. Within each panel, the $D=2.3$ models are higher because the radiation, both scattered and stellar, can escape more easily from the more strongly clumped structure. The lines show uniform models, 


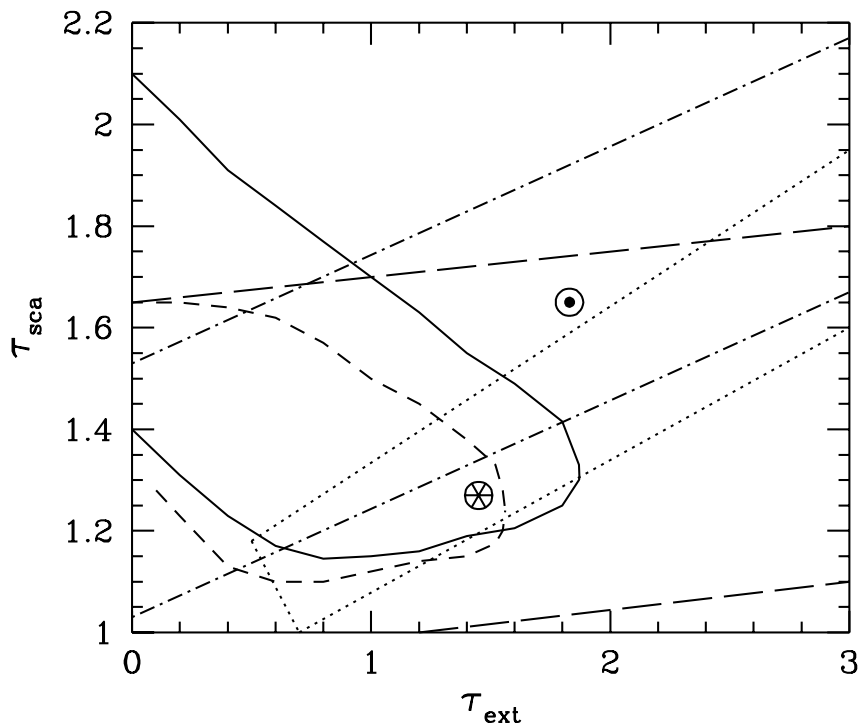

FIG. 3.- General areas of the ( $\left.\tau_{\mathrm{ext}}, \tau_{\mathrm{sca}}\right)$ plane that are covered by points from various hierarchical models. Axes are as in Figs. 1 and 2. Values of $\left(a, g, D, \tau_{0}\right)$ are $(0.8,0.6,2.3,0.5)$ (solid line); $(0.8,0.6,2.3,0.5)$, with $33 \%$ of dust mass in constant density (short-dashed line); $(0.6,0.6,2.6,2)($ dotdashed line); $(0.6,0.6,2.6,2)$, with constant density component (dotted line); and $(0.8,0.95,2.3,4)$ (long-dashed line). Circled points mark observations for NGC 7023, as in Figs. 1 and 2. We see that a wide variety of hierarchical models can cover the observations.

with albedos marked in Figure $4 a$. The uniform model appropriate to the actual albedo assumed in the models $(a=0.6)$ is just below the bottom of the figure. The dashed line is the uniform model with $a=0.5$ and $g=0.4$, instead of 0.6. The differences are not large in comparison with the

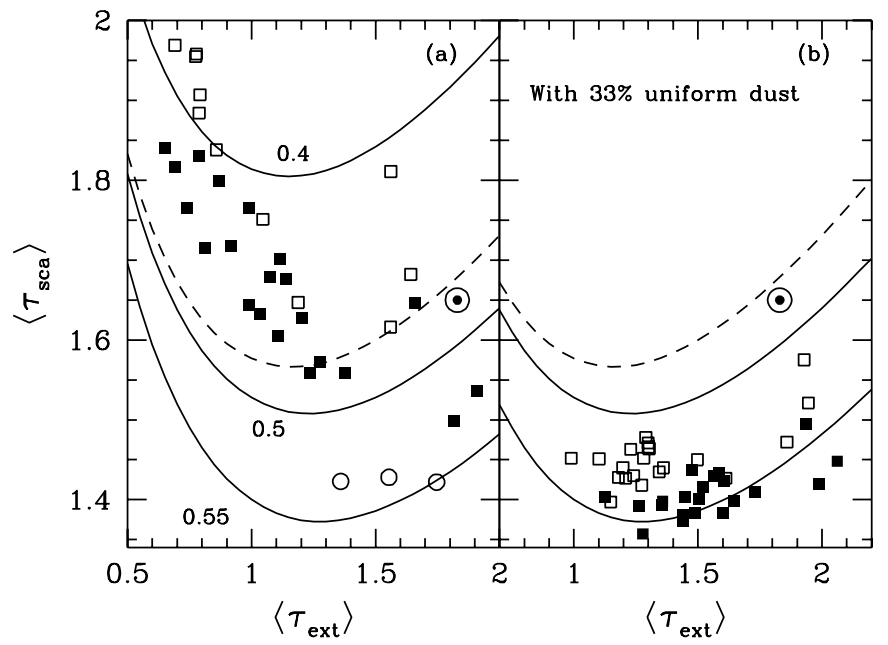

FIG. 4.-Optical depths for scattering and stellar radiation for various hierarchical models, averaged over viewing angles. All have $(a, g$, $\left.\tau_{0}\right)=(0.6,0.6,2)$. Each square represents a different arrangement of dust clumps. (a) Models with no uniform component; (b) models with $33 \%$ of the dust in a uniform component. Filled squares, $D=2.6$; open squares, $D=2.3$; solid lines, relationship for spatially uniform dust, with albedo as marked in $(a)$ and $g=0.6$; open circles in ( $a$ ), dust clumped in either of two densities, with parameters similar to those in Witt \& Gordon (2000), $a=0.6, g=0.6$, and different random spatial arrangements. The extremes of many different spatial arrangements are shown, along with a typical case. The dashed lines show uniform models with $a=0.5$ and $g=0.4$, instead of 0.6 . We see that the other parameters are more important than $g$. effects of the other parameters. The effects on hierarchical models of changing $g$ are similar.

Perhaps the most striking difference between the two panels is the lower values of $\left\langle\tau_{\text {sca }}\right\rangle$ for hierarchical models with $33 \%$ of the dust in a uniform component. The increase of scattering from the dust between the clumps causes the decrease in $\left\langle\tau_{\text {sca }}\right\rangle$ and greatly reduces the differences between models with different spatial distributions of dust. In either panel, the points from various viewing angles of the two values of $D$ are completely intertwined. Reflection nebulae are poor diagnostics of $D$, as well as of other properties of the ISM.

The three circles in Figure $4 a$ represent two-phase models with three values of the initial seed. Two of these values show the extrema in $\left(\left\langle\tau_{\text {ext }}\right\rangle,\left\langle\tau_{\text {sca }}\right\rangle\right)$ among the 21 initial seeds that we tested. The tightness of the mean optical depths of the two-phase models shows the importance of hierarchical geometry, as opposed to simple two-phase clumps. The $\left\langle\tau_{\text {sca }}\right\rangle$-values of the two-phase models are similar to those of the hierarchical models with uniform dust. The contrast of both with purely hierarchical models illustrates the importance of dust-free regions (in real space, possibly caused by extensions of hot, low-density material into the nebulae).

Figure 4 shows what we meant by saying that Figure 1 was produced by a typical hierarchical model, one with a typical $\left\langle\tau_{\text {ext }}\right\rangle$. Models with large $\left\langle\tau_{\text {ext }}\right\rangle$ have the star embedded within dusty material, and a relatively low central dust density leads to a small $\left\langle\tau_{\text {ext }}\right\rangle$.

Differences among models, and errors arising from interpreting reflection nebulae with uniform models, increase with optical depth. Figure 5 uses the same parameters as Figure $4(a=0.6 ; g=0.6)$, except that $\tau_{0}=4$, instead of 2 . The open squares are for various initial dust distributions, with $D=2.3$ and $33 \%$ of the density uniform, and the filled squares the same, but with $D=2.6$. The open triangles are for a purely hierarchical density with $D=2.3$ and the filled triangles the same, but with $D=2.6$. The circles enclose the entire range of our two-phase models.

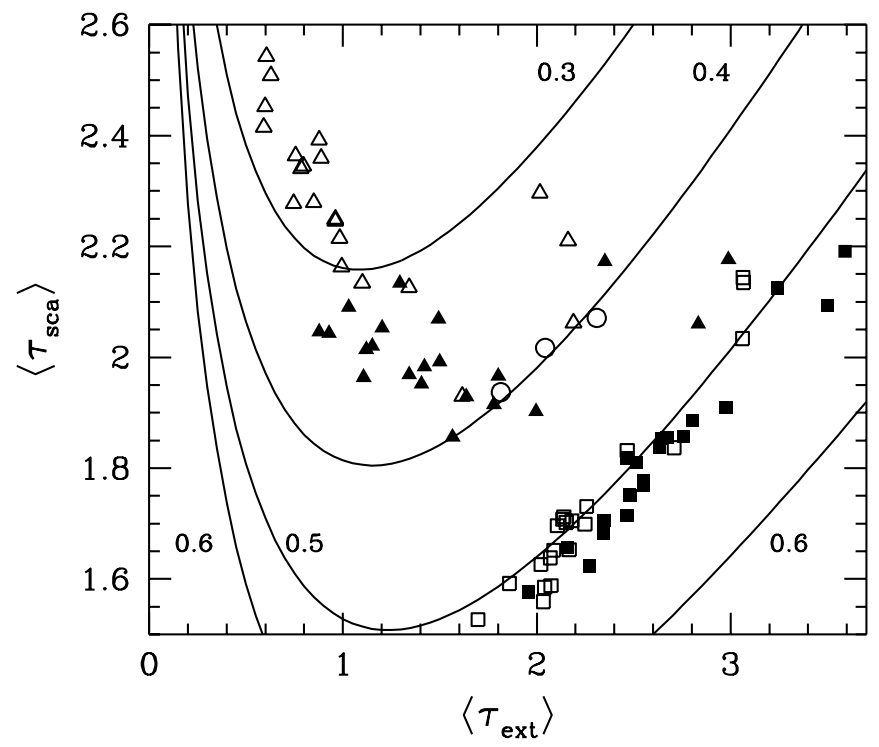

FIG. 5.-Same as Fig. $4 b$, except that $\tau_{0}$ is 4 , instead of 2. Open triangles: purely hierarchical models (no uniform density), with $D=2.3$; filled triangles: purely hierarchical models with $D=2.6$. Solid lines are for spatially uniform dust, with $a$ as marked and $g=0.6$. 
Figure 5 shows an important result: optically thick hierarchical models can show very modest values of $\left\langle\tau_{\text {ext }}\right\rangle$, but interpreting the nebula with uniform models will likely result in a severe underestimate of the albedo unless there is material between the clumps to scatter light efficiently. For the purely hierarchical models with $D=2.3$ (open triangles), there is very little scattered light between the clumps (i.e., large $\tau_{\text {sca }}$ ) and limited stellar extinction, resulting in albedos predicted from uniform models that are as low as 0.17. The spread among the two-phase models (circles) is relatively small because at large optical depth they become statistically uniform. Since they are clumpy, the uniform-model albedos they suggest are significantly lower than the 0.6 used in the actual two-phase models.

The squares in Figure 5 represent models in which 33\% of the dust mass is in a constant density, so that the minimum optical depth is $4 / 3$. This addition greatly increases the scattered flux and makes the difference between $D=2.3$ and $D=2.6$ almost unimportant. The albedo that would be derived from uniform models is increased to $\sim 0.5$ over the purely hierarchical models, still short of the correct value of 0.6 .

The spread of the $\left\langle\tau_{\text {ext }}\right\rangle$ and $\left\langle\tau_{\text {sca }}\right\rangle$ in Figures 4 and 5 show that the specification of $\tau_{0}$ and $D$ (along with grain properties) does not specify the scattered and stellar fluxes from reflection nebulae in even a statistical sense. By contrast, $D$ constrains the statistical properties of the projected brightness distribution for both line and continuum emission from the gas. The large variations in properties of reflection nebulae with viewing angle come about because the radiation arises from the geometry of the statistical distribution of the ISM relative to a point source rather than to itself. Emissions from gas involve an integration over larger regions of more equal weight.

It is unrealistic to suppose that reflection nebulae are spherical with a central exciting star. We have considered only this geometry because we feel that it provides rather general results for strongly forward-throwing scattering (say, $g \gtrsim 0.5$ ). In this case, the dust on the near side of the nebula is likely to provide most of the scattered light. We consider our models as representing only this near-side dust.

\section{DISCUSSION}

\subsection{Reflection Nebulae}

Our hierarchical models, with their overall spherical distribution of clumps, are primarily aimed at interpreting reflected light from reflection nebulae illuminated by an exciting star. We discuss reflection of the diffuse Galactic radiation field from dusty globules at the end of this section.

We now examine the limits on $a$ imposed by the amount of scattered light observed in reflection nebulae. Since real nebulae show the illuminated cloud as it is viewed from only one direction, we must consider the spread of $\left(\tau_{\text {ext }}, \tau_{\text {sca }}\right)$ from any particular dust distribution. We seek $a_{\max }$ and $a_{\text {min }}$, the minimum and maximum values of $a$ that can fit the observations.

For illustrative purposes, we consider the $1300 \AA$ observations of NGC 7023, with $50 \%$ of the stellar extinction assumed to be within the nebula (Witt et al. 1993; circled dot in Figs. 1 and 2). In Figure 1 it lies in the middle of the envelope of the $\left(\tau_{\text {ext }}, \tau_{\text {sca }}\right)$ points from various viewing angles. If we decreased the $a$ of the model, the scattered flux at each direction would become fainter and $\tau_{\text {sca }}$ larger. The stellar fluxes would be unchanged because they depend only on extinction, not scattering. Thus, the pattern of points would move up in the diagram, and the point could no longer be fitted. To find the $a_{\text {min }}$ for NGC 7023, we must vary $\tau_{0}$ and initial seeds (dust distributions) and find the minimum $a$ for which the envelope of individual points lies just above the observation. A similar procedure leads to $a_{\text {max }}$. Of course, it is improbable that the actual $a$ is as low as $a_{\text {min }}$, because the observed $\left(\tau_{\text {ext }}, \tau_{\text {sca }}\right.$ ) would probably not lie at the edge of the ( $\left.\tau_{\text {ext }}, \tau_{\text {sca }}\right)$ distribution as seen from a random direction.

We have performed this exercise and found the following.

1. All of our models required an albedo of $\gtrsim 0.5$ to fit the lower point of NGC 7023. Varying parameters and geometries to obtain the absolute minimum $a$ ( 0.46 ; see below) produced only changes in details. This result is robust because the observed $\tau_{\text {ext }}$ is near the most efficient value for producing scattered light, as suggested by the uniform models (see the minima of $\tau_{\text {sca }}$ in the curves in Fig. 1).

2. The scattered light is near its maximum when $\tau_{0} \sim 2$, where the mean $\tau_{\text {ext }}$ is about unity, so $a_{\text {min }}$ is best estimated for our hierarchical models near this value. The maximum scattered light for uniform models occurs at $\tau_{0} \sim 1.1-1.4$, increasing slowly with the albedo.

3. The lowest $a_{\min }$ we found for the circled dot in Figure 1 is 0.48 . It occurs for $\tau_{0} \sim 2, D=2.6$, and a particular seed whose $\left(\left\langle\tau_{\text {ext }}\right\rangle,\left\langle\tau_{\text {sca }}\right\rangle\right)$ point is low in Figure 4 . The effects of $g$ are minimal on the locus of the lower envelope of the individual points; the minimum $a$ is achieved for $g \geq 0.5$. We tried such a wide range of models that we doubt that there is an $a_{\text {min }}$ drastically lower.

4. No useful estimate of $a_{\max }$ is possible, because inefficient scattering can be produced by extreme clumping (as small a $D$ as allowed) and with no uniform component. For $D=2.3$, an $a \sim 1$ is needed to fit NGC 7023 for some distributions of clumps. For $D=2.6, a_{\max }$ is about 0.8 . The dust geometry that produced $a_{\text {min }}$ did not allow $a$ to be above 0.6 , but it was chosen to have especially efficient scattering. Alternatively, very optically thick models that have low stellar extinctions, as seen from some viewing angles, can fit observed fluxes with very high albedos. For instance, we have fitted the NGC 7023 point in Figure 1 with two-phase models, $\tau_{0}=8$, and $a=0.85$. We have no doubt that many optically thick hierarchical models could fit the observations as well. Perhaps such extreme models could be ruled out with FIR fluxes, although the low absorption tends to compensate for the high optical depth. Furthermore, the FIR arises from dust surrounding the star in all directions, while the scattering is mainly from dust close to the line of sight. In any case, these models are hardly reasonable on physical grounds.

Voyager 2 and HUT observed NGC 7023 at $1000 \AA$ (Witt et al. 1993). Uniform models predict $a \sim 0.45$ if $40 \%$ of the stellar extinction occurs within the nebula. Hierarchical models can fit the observation (not plotted, but in the upper right corner of Figs. $1-3$ ) with $a=0.8$ for $\tau_{0} \gtrsim 4$. Of course, we make no claim that such a high $a$ is correct. On the other hand, we can fit the point with $a=0.4$, lower than the uniform models. Once again, we see that the observations are subject to a very ambiguous interpretation. 


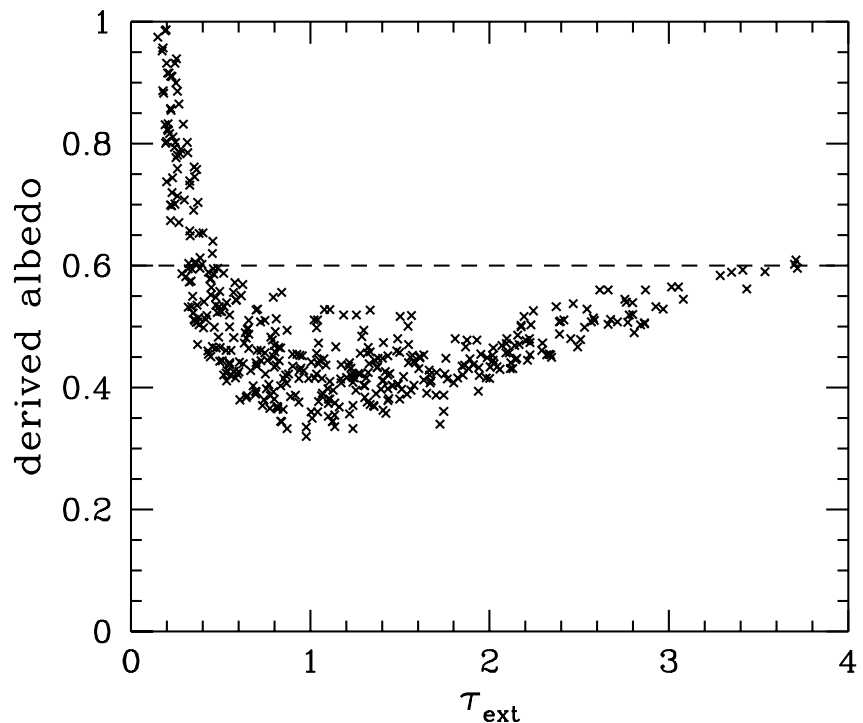

FIG. 6.-Albedo derived from uniform models, using the scattered flux and extinction of a typical purely hierarchical model as seen from various viewing angles, vs. stellar extinction. Parameters are given in the text. The "true" albedo used in the generating model, 0.6, is marked by the dashed line.

Burgh, McCandliss, \& Feldman (2002) used a uniform model for NGC 2023, with $\tau_{\text {ext }}=2.4$. They derived an albedo of $0.39_{-0.05}^{+0.12}$ at $1345 \AA$. Our hierarchical model shown in Figure 1, with $a=0.6$, could accommodate their observations, and others that scatter light inefficiently could have $a \sim 1$.

Figure 6 shows the albedos derived from uniform models, using the scattering and extinctions from the hierarchical model $\left(a, g, D, \tau_{0}\right)=(0.6,0.6,2.3,1)$, plotted against the stellar extinctions at each viewing angle. The albedo used to generate the scattered fluxes and extinctions, 0.6, is marked by the dashed line. The distribution of points for the hierarchical model with $\tau_{0}=2$ is virtually identical. We see that the minimum $a$ derived from uniform models is $\sim 0.32$; the average for $0.6 \leq \tau_{\text {ext }} \leq 1.6$ is $\sim 0.42$. At moderate extinction, leading to bright scattered flux, uniform models underestimate $a$. At small $\tau_{\text {ext }}$ uniform models overestimate $a$. A hierarchical model in which the star happens to be within a clump has smaller errors. Adding a constant density decreases the errors at $\tau_{\text {ext }} \sim 1$, so that the average $a$ is $\sim 0.5$. Of course, there is no error in the unrealistic case of the density being uniform throughout.

An important question is, how reliably can we determine $a(\lambda)$ from observations of a particular nebula covering a significant range in wavelengths, implying differing opacities or optical depths? In this case, we might hope to find relative optical properties of the grains accurately, even with uniform models. Figure 7 shows the results for two wavelengths for which the extinctions (both $\tau_{0}$ and $\tau_{\text {ext }}$ ) differ by a factor of 2: for example, 1000 and $1540 \AA$, if the extinction law is the Galactic average (Cardelli, Clayton, \& Mathis 1989) and $R_{V}=A(V) /[A(B)-A(V)]=3.1$. The figure displays the ratio of $a\left(\tau_{0}=2\right)$ to $a\left(\tau_{0}=1\right)$, both albedos derived from uniform models, for the same hierarchical model as shown in Figure 1. The abscissa in the figure is $\tau_{\text {ext }}$ for the lower extinction. All points in the figure were computed with $a=0.6$, so the "true" value of the albedo ratio is

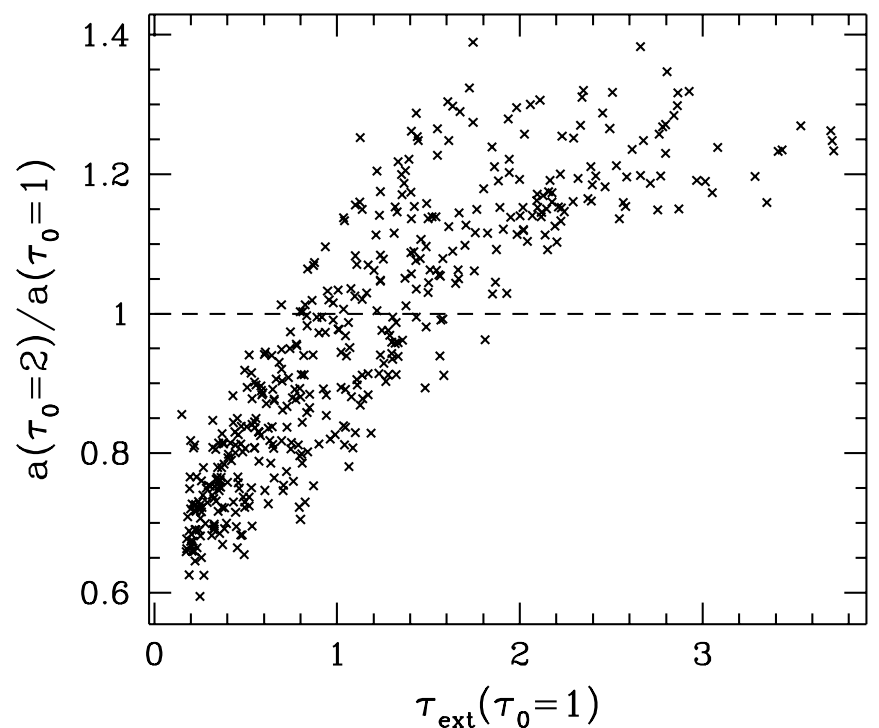

FIG. 7.-Test of whether relative values of the albedo can be derived from observations of a nebula at two different wavelengths or optical depths, with a given arrangement of dust relative to the central star. Here we compare averaged optical depths, $\tau_{0}$, of 2 and 1 , for a particular hierarchical model. Since only $\tau_{0}$ is varied, the geometry of the dust is the same. The scattering and stellar fluxes at various viewing angles were interpreted with uniform-density models. The ratio of the derived albedos is plotted against the smaller stellar extinction. The dashed line gives the "true" value of unity, since all points were derived with $a=0.6$.

unity. We see that uniform models have difficulty in predicting even relative values of $a(\lambda)$, since the spread in $a\left(\tau_{0}=2\right) / a\left(\tau_{0}=1\right)$ is $\sim 40 \%$. The figure gives the impression that if $\tau_{\text {ext }}$ is $\geq 1.5$, uniform models would derive a higher albedo at short wavelengths (say, $1000 \AA$ ) than at longer (1540 $\AA$ for a factor of 2 in the extinction). For NGC 7023 the opposite is found to be the case (Murthy et al. 1993; Witt et al. 1993). From Figure 7 we would conclude that $a(1000 \AA)<a(1540 \AA)$ is robust, since Witt et al. (1993) suggest $\tau_{\text {ext }}(1540 \AA) \sim(1.5-2)$. However, models with the star embedded in a clump have the albedo ratios shift from mostly less than 1 to mostly greater than 1 at $\tau_{\text {ext }}(1540 \AA) \sim 2.5$, considerably greater than shown in the figure. These models would produce an illusory result $a(1000 \AA)<a(1740 \AA)$.

The basic problem is that the importance of a clump depends upon its optical depth. If a clump becomes optically thin, its existence no longer very significant to the radiative transport. Similarly, increasing the optical depth of an optically thick clump has a limited effect on the radiation. While the density structure within the ISM is independent of the wavelength, its geometry as regards radiation transfer is not. Since the clumping produces the large spread in $\tau_{\text {sca }}(\theta, \phi)$, it is not surprising that there is a wide spread in the importance of the clumps among various viewing angles.

The problems of determining the variation of albedo at various wavelengths are small if the ratio of optical depths is close to unity. Statements that the albedo is lower at the $2175 \AA$ feature than on either side of it (see, e.g., Witt et al. 1992) seem robust, besides being completely plausible physically. At nearby wavelengths $A(\lambda) / N(\mathrm{H})$, the extinction per $\mathrm{H}$ nucleus, can be the same at three wavelengths, and com- 
parison of the relative albedos between those wavelengths is completely safe.

We have seen that several properties of reflection nebulae change if the exciting star is embedded within a rather dense clump of dust. There is an increase in the scattered light; the lowest values of $\left\langle\tau_{\text {sca }}\right\rangle$ for purely hierarchical models in Figure $4 a$ (i.e., the large amounts of scattered light for the purely hierarchical models) belong to such cases. The central clump ensures that the angle-averaged extinction, $\left\langle\tau_{\mathrm{ext}}\right\rangle$, is larger than for most models with the same $\tau_{0}$ (i.e., the points in Fig. $4 a$ tend to have large $\left\langle\tau_{\text {ext }}\right\rangle$ if $\left\langle\tau_{\text {sca }}\right\rangle$ is small). Figure $4 b$ shows that the addition of a constant-density component makes the effects of a central clump rather unimportant, because the radiation scattered by the uniform dust dominates. Another effect of a star being embedded in a dense clump is that the errors of the albedo derived from observations of the same object at different wavelengths, illustrated in Figure 6, are reduced because more of the scattering arises from the central clump. For such a clump, the opacity, but not the geometry, changes with wavelength. Finally, there would tend to be more FIR radiation from an embedded star because the grains are relatively close to the star so they are absorbing a relatively large stellar flux, and the mean temperature of the grains would be relatively large for the same reason.

Galactic radiation scattered by isolated globules seen away from the Galactic plane provides another diagnostic of grains (Mattila 1970). Witt, Oliveri, \& Schild (1990) have analyzed such a globule and give references to other examples and discussion of the process. The density is concentrated toward the center of such globules. The reflected intensity rises outward from the center, peaks, and falls off to the sky brightness at the edge, showing that the scattering is forward-throwing. The reflected intensity can be related to the incident radiation predicted for both stars and the DGL out of the Galactic plane. Results were that at 4700 A grains are forward-throwing $(g \sim 0.8)$ and very efficient at scattering $(a \sim 0.8)$. The globule was modeled with a very centrally condensed density distribution that is smooth and spherically symmetric, as seemed completely appropriate at the time. Since turbulence driving clumps in the diffuse ISM may decay when driving into a steepening density gradient, perhaps the ISM within globules is relatively free from clumping. We cannot assess the uncertainties in the grain properties derived from globules until we know more about the clumping within them. The major uncertainty would then be the prediction of the radiation incident upon them from the hierarchically clumped Galaxy.

\subsection{The Diffuse Galactic Light}

There is no doubt about the applicability of hierarchical clumping to the DGL. Observations such as those at $1740 \AA$ (Schiminovich et al. 2001) are based on large portions of the sky. We have seen that uniform spherical models of reflection nebulae underestimate $a$ if applied to optically thick situations (large $\tau_{0}$, but not necessarily large $\tau_{\text {ext }}$ ). Our reflection nebula models are not ideally suited for interpreting the DGL in the UV, since distributions of the dust and exciting stars are skewed toward the Galactic plane. However, our results have strong implications as regards the interpretation of the DGL as discussed in Henry (2002, hereafter H02). In that paper, it was concluded that $a \lesssim 0.1$ for $\lambda \lesssim 1400 \AA$.
$\mathrm{H} 02$ performed radiative transport calculations by using $B$ and $V$ magnitudes and the spectral type for each star in the entire Hipparcos Input Catalogue. The reddening, $E(B-V)$, follows from $(B-V)$ and spectral type. The extinction $A_{\lambda}$ is $E(B-V) R_{V}\left(A_{\lambda} / A_{V}\right)$, where $R_{V}$ is usually taken to be 3.1. The $\tau_{\text {ext }}$ for each star is $A(\lambda) / 1.086$.

The heart of $\mathrm{H} 02$ is its unique treatment of radiative transfer. The scattering is taken to be

$$
\begin{aligned}
& \exp \left(-\tau_{\text {sca }}\right)=a\left(1-e^{-\tau_{\text {ext }}}\right) \\
& \quad \times \exp \left\{-(1-a)\left[\tau_{\text {ext }}+\ln \left(1+e^{-\tau_{\text {ext }}}\right)-\ln 2\right]\right\},
\end{aligned}
$$

independent of the geometry. However, Witt, Friedmann, \& Sasseen (1997) have shown from clumpy models that the distribution of cloud optical depths can strongly affect the interstellar radiation field.

At low optical depths, the $\mathrm{H} 02$ relation between $\tau_{\text {ext }}$ and $\tau_{\text {sca }}$ approaches the uniform model. The dotted lines in Figure 1 show the $\mathrm{H} 02$ models for three albedos identified by the nearby solid lines. We see that the H02 assumption predicts more scattered light than uniform models, and a lower albedo follows.

For $\lambda \lesssim 2000 \AA$, the $\tau_{\text {ext }}$ for most hot stars is probably rather large, since the mean $A(V)$ is $\sim 1 \mathrm{mag} \mathrm{kpc}^{-1}$ and that for the UV is $3-5$ times larger. For $\tau_{\mathrm{ext}}=1.5$, Figure 1 shows that the $\mathrm{H} 02$ model with $a=0.6$ predicts a scattered flux $\sim \exp (0.4)=1.5$ times larger than that predicted by the "typical" hierarchical model, chosen for illustrative purposes only. The conclusion of $\mathrm{H} 02$ that $a(1000 \AA)$ is very low ("perhaps 0.1 ") is perhaps understandable. Such a low albedo is not compatible with the observations of NGC 7023 by Witt et al. (1993), in which the scattered flux was larger than the stellar, or Murthy et al. (1993), which suggests that $a(1150 \AA) \sim 0.55$ if $40 \%$ of the extinction is within the nebula, as assumed by Witt et al. (1992).

Murthy, Henry, \& Holberg (1991) used Voyager 2 to search for DGL for $\lambda \leq 1300 \AA$. They failed to detect any, possibly confirming the $\mathrm{H} 02$ picture. Our reflection nebula results commonly show that the same nebula can show very low and rather high scattered fluxes when seen from different angles. We strongly suspect that the same property applies to the DGL. Our general result from hierarchical models is that photons, especially the unscattered ones coming directly from the star, can leak out of dust distributions far more easily than either uniform-density or H02 models would predict, so a dark sky does not necessarily imply a low albedo.

\section{FINAL REMARKS AND SUMMARY}

We have arrived at a pessimistic but, we feel, realistic assessment of the ability of reflection nebulae illuminated by embedded stars to serve as diagnostics of interstellar grain properties. It is better to realize what we do not know than to believe that we know more than we do! Even if the scattered light from a reflection nebula and the extinction suffered by its exciting star are observed perfectly, the geometry of the dust is vital to the interpretation of the observations. Hierarchical clumping, "clumps within clumps within clumps," has a very large impact on the interpretation of both reflection nebulae and the DGL as compared to smooth density structures. Hierarchical structures, demonstrated by observations in $\mathrm{CO}, 100 \mu \mathrm{m}$, various other molecules, and $\mathrm{H} \mathrm{I}$, make any determination of the optical 
properties of grains from reflection nebulae very difficult. Our models had plausible power spectra for the autocorrelation function of the projected densities, with an exponent of the power of $\sim-3$, unlike either uniform models or those that are clumped on a single scale (exponent $\sim 0$ ). The exact geometry of the dust is very difficult to determine because it depends on the placement of hierarchical clumps of dust around the star.

We have made no interpretations of any reflection nebulae. It is now relatively easy to use Monte Carlo transfer codes to predict the scattering (and polarization) for objects with a known geometry. Inverting observed intensities to determine the correct geometry, however, is very difficult, even with observations with good spatial resolution taken in multiple wavelengths. Our hierarchical models have been schematic and do not begin to exhaust the possibilities of the real geometry of any reflection nebula. Observations in the infrared and line emissions with good spatial resolution may eventually put useful limits on the actual geometry of the gas and dust, but the problem of inverting the data will always be difficult.

An interesting possibility is that isolated Bok globules may not be strongly clumped, so reflection of Galactic light from them can diagnose grain properties. The penetration of turbulence into them might be weaker than its percolation through larger structures. Nonclumpy globules can serve to diagnose grains because they scatter incident Galactic radiation (see Witt et al. 1990 for references and an explanation).

Each of our hierarchical models shows a very wide range of extinction optical depths $\left(\tau_{\text {ext }}\right)$ and scattering $\left(\tau_{\text {sca }}\right)$ when the nebula is viewed from various directions. The $\tau_{\text {ext }}$ and $\tau_{\text {sca }}$ observed for a reflection nebula represent a view of the dust from one direction. There is a very wide range of optical properties $(a, g)$ that can fit a given observation. With one or more hierarchical models we can fit the best-observed UV reflection nebula, NGC 7023 , with albedos $\geq 0.5$ over the range of $g$ that we tested (0.6-0.85) and with a variety of averaged optical depths through the model.

In general, reflection nebulae have substantial optical depths $\left(\tau_{\text {ext }} \sim 1-3\right)$; otherwise, the scattered fluxes are too faint. At substantial optical depths, uniform models can underestimate the albedo rather severely because they overestimate the scattered light. Unless the star happens to be embedded within a dense clump, radiation can leak out of hierarchical clumps much more easily than from uniform dust.

The diffuse Galactic light (DGL) has been interpreted (H02) with a recipe that predicts much more scattering from each star than our models, resulting in a low estimate of the UV albedo. Murthy et al. (1991) failed to detect UV DGL, but our fractal models often have many directions from which the star and scattered light are very faint. Thus, we do not believe that the faintness of the DGL in particular directions necessarily signifies a low albedo.

The determination of both $a$ and $g$ depends upon the variation of reflected intensity across the face of a centrally illuminated reflection nebula, while we have only considered the flux of the scattered light. We feel that if the flux is as weakly constrained as our models show, the intensity will be similarly subject to variation because of the unknown placement of the star relative to the surrounding dust.

Even the relative variation of albedo with wavelength is difficult to determine from reflection nebulae if there are significant changes in opacity among the wavelengths. The problem is that the geometry is not really the same when the opacity changes. As opacity per $\mathrm{H}$ atom increases, optically thin clumps become thick and scatter light with a different geometrical arrangement, so the geometry of the nebula depends on wavelength.

Our overall assessment is that the optical properties of grains are probably as well constrained by theory as by observations. This statement is made in spite of well-known uncertainties in the theory of interstellar grains. These uncertainties are major: whether typical large grains are chemically homogeneous (e.g., silicate or carbonaceous) or composite, whether grains contain voids, or if grains have very loose ("fractal") structure. However, theory does not permit the large range of possible albedos provided by reflection nebulae. At first glance, various theories seem quite different. Weingartner \& Draine (2001) have polycyclic aromatic hydrocarbon (PAH) molecules, small grains, and large grains of silicate or graphite. Li \& Greenberg (1997) have PAHs, small grains, and large grains with silicate cores and organic refractory mantles. Mathis (1996) has composite grains with silicates, carbon, and vacuum intermixed within the same grain, plus small graphitic particles that mimic PAHs. Draine \& Lee (1984) have silicate and graphite grains with a truncated power-law distribution of sizes. Each size distribution is different. They all predict roughly the same $a(\lambda)$ because the response of the grains to radiation is set by fitting the well-observed interstellar extinction law $\tau_{\text {ext }}(\lambda)$, and cosmic abundances (although somewhat controversial themselves) constrain the materials. For instance, the values predicted for $a(1430 \AA)$ are $0.40,0.30,0.37$, and 0.40 for Weingartner \& Draine (2001), Li \& Greenberg (1997), Mathis (1996), and Draine \& Lee (1984), respectively. The $A(V)$ values are $0.65,0.63,0.53$, and 0.57 .

Fortunately, optical depths and column densities derived from comparisons of absorption and scattering along the same very narrow beam (i.e., toward a star) are not affected by the microstructure within the ISM. These measurements include most determinations of depletions of various ions in the ISM, as well as the wavelength dependence of the interstellar extinction law. Other implications for the hierarchical nature of dust clumping include a greatly increased penetration of radiation into molecular clouds and photodissociation regions (PDRs).

We would like to thank Professor A. Witt for constructive comments on a draft of this paper that improved it considerably. B. W. and K. W. acknowledge financial support from NASA's Long Term Space Astrophysics Research Program, NAG5-6039 (K. W.), NAG5-8412 (B. W.), the National Science Foundation, AST99-09966 (B. W. and K. W.), and a PPARC Advanced Fellowship (K. W.). We appreciate the constructive and prompt comments of two anonymous referees. This research has benefited from the NASA Astrophysics Data System and the SIMBAD database. 


\section{REFERENCES}

Abergel, A., Boulanger, F., Delouis, J. M., Dudziak, G., \& Steindling, S. 1996, A\&A, 309, 245

Andrews, S. M., Meyer, D. M., \& Lauroesch, J. T. 2001, ApJ, 552, L73

Boissé, P. 1990, A\&A, 228, 483

Burgh, E. B., McCandliss, S. R., \& Feldman, P. D. 2002, ApJ, in press

Cardelli, J. A., Clayton, G. C., \& Mathis, J. S. 1989, ApJ, 345, 245

Chappell, D., \& Scalo, J. 2001, ApJ, 551, 712

Code, A. D. 1973, in IAU Symp. 52, Interstellar Dust and Related Topics,

ed. J. M. Greenberg \& H. C. van de Hulst (Dordrecht: Reidel), 505

Crovisier, J., \& Dickey, J. M. 1983, A\&A, 122, 282

Draine, B. T., \& Lee, H. M. 1984, ApJ, 285, 89

Elmegreen, B. G. 1997, ApJ, 477, 196

Elmegreen, B. G., \& Falgarone, E. 1996, ApJ, 471, 816

Faison, M. D., \& Goss, W. M. 2001, AJ, 121, 2706

Falgarone, E., Panis, J.-F., Heithausen, A., Pérault, M., Stutzki, J., Puget,

J.-L., \& Bensch, F. 1998, A\&A, 331, 669

Green, D. A. 1993, MNRAS, 262, 327

Heiles, C. 1997, ApJ, 481, 193

Heithausen, A., Bensch, F., Stutzki, J., Falgarone, E., \& Panis, J.-F. 1998, A\&A, 331, L65

Henry, R. C. 2002, ApJ, 570, 697 (H02)

Henyey, L. G., \& Greenstein, J. L. 1941, ApJ, 93, 70

Jenkins, E. B., \& Tripp, T. M. 2001, ApJS, 137, 297

Knauth, D. C., Federman, S. R., Pan, K., Yan, M., \& Lambert, D. L. 2001, ApJS, 135, 201

Li, A., \& Greenberg, J. M. 1997, A\&A, 323, 566

Martini, P., Sellgren, K., \& DePoy, D. L. 1999, ApJ, 526, 772

Mathis, J. S. 1996, ApJ, 472, 643
Mattila, K. 1970, A\&A, 9, 53

Moore, E. M., \& Marscher, A. P. 1995, ApJ, 452, 671

Murthy, J., Dring, A., Henry, R. C., Kruk, J. W., Blair, W. P., Kimble, R. A., \& Durrance, S. T. 1993, ApJ, 408, L97

Murthy, J., Henry, R. C. \& Holberg, J. B. 1991, ApJ, 383, 198

Norman, C. A., \& Ferrara, A. 1996, ApJ, 467, 280

Press, W. H., Teukolsky, S. A., Vetterling, W. T., \& Flannery, B. P. 1992, Numerical Recipes in $\mathrm{C}++$ (2d ed.; New York: Cambridge Univ. Press)

Schiminovich, D., Friedman, P. G., Martin, C., \& Morrissey, P. F. 2001, ApJ, 563, L161

Sellgren, K., Werner, M. W., \& Dinerstein, H. L. 1992, ApJ, 400, 238

Stanimirović, S., Staveley-Smith, L., Dickey, J. M., Sault, R. J., \& Snowden, S. L. 1999, MNRAS, 302, 417

Vogelaar, M. G. R., \& Wakker, B. P. 1994, A\&A, 291, 557

Weingartner, J. C., \& Draine, B. T. 2001, ApJ, 548, 296

Welty, D. E., \& Fitzpatrick, E. L. 2001, ApJ, 551, L175

Westpfahl, D. J., Coleman, P. H., Alexander, J., \& Tongue, T. 1999, AJ, 117,868

Witt, A. N., Friedmann, B. C., \& Sasseen, T. P. 1997, ApJ, 481, 809

Witt, A. N., \& Gordon, K. D. 1996, ApJ, 463, 681 2000, ApJ, 528, 799

Witt, A. N., Oliveri, M. V., \& Schild, R. E. 1990, AJ, 99, 888

Witt, A. N., Petersohn, J. K., Bohlin, R. C., O'Connell, R. W., Roberts, M. S., Smith, A. M., \& Stecher, T. P. 1992, ApJ, 395, L5

Witt, A. N., Petersohn, J. K., Holberg, J. B., Murthy, J., Dring, A., \& Henry, R. C. 1993, ApJ, 410, 714

Wood, K., \& Reynolds, R. J. 1999, ApJ, 525, 799 